\title{
Finite Element Analysis of Tsunami Propagation
}

\author{
Hashentuya \\ Graduate School of Environmental Science \\ Okayama University \\ Okayama City, Japan \\ E-mail:dev422252@s.okayama-u.ac.jp
}

\author{
Watanabe \\ Graduate School of Environmental and Life Science, \\ Okayama University \\ Okayama City, Japan \\ E-mail:watanabe@ems.okayama-u.ac.jp
}

\author{
Kazuhiro Yamamoto \\ Graduate School of Environmental Science \\ Okayama University \\ Okayama City, Japan \\ E-mail:dev421102@s.okayama-u.ac.jp
}

\begin{abstract}
A tsunami generated in the North Pacific is simulated numerically. A system of partial differential equations derived from equations of a fluid dynamics were solved numerically by a finite element method. Depth data originally given in terms of longitude and latitude are transformed to projected coordinates by the Gauss-Kruger projection. The governing equations were spatially discretized by a finite element method. A resultant system of ordinary differential equations were solved numerically using a standard ODE solver. A numerical result is verified in comparison with a previous result.
\end{abstract}

Keywords- Tsunami propagation; Finite element method; Numerical solution; PECE Mode

\section{INTRODUCTION}

The Nankai Trough off coast of Japan is a major source of earthquakes and tsunamis in the North Pacific Ocean. An earthquake of 7.5-magnitude generated a tsunami, called "Hyuganada tsunami" on April 1, 1968 in the area [1]. In this study the propagation of the Hyuganada tsunami is simulated with novel techniques.

Techniques based on shallow water equations have been proposed to analyze tsunami waves [2-4]. The system of governing partial differential equations are spatially discretized by a finite element method, and reduced to a system of ordinary differential equations to which a standard ODE solver is applicable .

\section{GOVERNING EQUATIONS FOR SIMULATION}

A system of partial differential equations (1) were solved numerically to simulate the propagation of tsunami.

$$
\begin{aligned}
& \frac{\partial M}{\partial t}+g(h+\zeta) \frac{\partial \zeta}{\partial x}=0 \\
& \frac{\partial N}{\partial t}+g(h+\zeta) \frac{\partial \zeta}{\partial y}=0 \\
& \frac{\partial \zeta}{\partial t}+\frac{\partial M}{\partial x}+\frac{\partial N}{\partial y}=0
\end{aligned}
$$

C 2013. The authors - Published by Atlantis Press
These equations are based on a continuity equation and equations of motion to analyze long wave [5]. Here, $g$ is the gravitational acceleration, $h(x, y)$ and $\varsigma(x, y, t)$ are the bottom topography of the sea and the water elevation, respectively, and $M(x, y, t)$ and $N(x, y, t)$ are obtained by integrating the $x$-component and the $y$-component of the velocity $u$ and $v$, respectively,

$$
M=\int_{-h}^{\zeta} u d z, \quad N=\int_{-h}^{\zeta} v d z
$$

Given a triangular mesh covering a domain in the $x y$ plain with $n$ nodes and $m$ elements, let $\Phi_{j}(x, y)$ be the basis function associate with the $i$ th node $\left(x_{i}, y_{i}\right)$ i.e., $\Phi_{j}(x, y)$ is a continuous function over the domain, a linear function of $x$ and $y$ over each element, its support is contained in the union of the elements which have the $j$ th node as one of its vertices, and satisfies

$$
\begin{aligned}
& \Phi_{i}\left(x_{j}, y_{j}\right)=\delta_{i j}= \begin{cases}1 & i=j \\
0 & i \neq j\end{cases} \\
& i=1,2, \cdots, n, \quad j=1,2, \cdots, n .
\end{aligned}
$$

Suppose that the functions $h(x, y), \varsigma(x, y, t), M(x, y, t)$, and $N(x, y, t)$ are approximated by a linear combination of the basis functions with unknown coefficients $h_{j}, \zeta_{j}(t)$,

$$
\begin{array}{r}
M_{j}(t), N_{j}(t): \\
M(x, y, t)=\sum_{j=1}^{n} M_{j}(t) \Phi_{j}(x, y) \\
N(x, y, t)=\sum_{j=1}^{n} N_{j}(t) \Phi_{j}(x, y) \\
\zeta(x, y, t)=\sum_{j=1}^{n} \zeta_{j}(t) \Phi_{j}(x, y) \\
h(x, y)=\sum_{j=1}^{n} h_{j} \Phi_{j}(x, y) .
\end{array}
$$


Substituting the expressions (4) into the system (1), and set $(x, y)=\left(x_{i}, y_{i}\right)$, and the system (4) becomes

$$
\begin{aligned}
\frac{d M_{i}}{d t} & =-g\left(h_{i}+\zeta_{i}\right) \frac{\partial \zeta}{\partial x} \\
\frac{d N_{i}}{d t} & =-g\left(h_{i}+\zeta_{i}\right) \frac{\partial \zeta}{\partial y} \\
\frac{d \zeta_{i}}{d t} & =-\frac{\partial M}{\partial x}-\frac{\partial N}{\partial y} .
\end{aligned}
$$

The spatial derivatives on the right-hand sides of the system (5) are evaluated at $(x, y)=\left(x_{i}, y_{i}\right)$, and can be approximated by their average over the elements that contain the node $\left(x_{i}, y_{i}\right)$ in common.

\section{VERIFICATION OF NUMERICAL METHOD}

\section{A. Description of the Model Problem}

In this section, our numerical techniques are tested against a model problem. The techniques described in the previous section are applied to a system of partial differential equations obtained from equations of the nonlinear shallow-water wave. The numerical solutions correspond to time dependent motions in a basin of the paraboloid of revolution. A characteristic feature of this problem is the moving shoreline. It must be determined as a part of the solution of an initial boundary value problem.

Exact solutions for nonlinear fluid motions with moving boundaries are quite rare. The motion is governed by the shallow-water wave equations and the shoreline is the moving boundary. Some exact solutions to the nonlinear shallow-water wave equations have been proposed [6]. Here an analytic solution with the planar surface is compared with a numerical solution. The water dynamics is governed by the shallow-water wave equations [6].

$$
\begin{gathered}
\frac{\partial u}{\partial t}+u \frac{\partial u}{\partial x}+v \frac{\partial u}{\partial y}-f v+g \frac{\partial h}{\partial x}=0, \\
\frac{\partial v}{\partial t}+u \frac{\partial v}{\partial x}+v \frac{\partial v}{\partial y}+f u+g \frac{\partial h}{\partial y}=0, \\
\frac{\partial h}{\partial t}+\frac{\partial}{\partial x}[u(D+h)]+\frac{\partial}{\partial y}[v(D+h)]=0,
\end{gathered}
$$

The variables $u$ and $v$ are the $x$ and $y$ componets of the velocity. The Coriolis Parameter, $f$, accounts the earth's rotation, and $\mathrm{g}$ is the gravitational acceleration. Equation (8) is the continuity equation.

The surface elevation, $\mathrm{h}$, is positive if it is above the equilibrium level, whereas the depth function, $D$, is positive below the equilibrium level. Thus, $D+h$ is the total depth of the fluid [6]. We assume $f=0$. The instantaneous shoreline is determined by the condition, $D+h=0$. We will consider the special condition $l=L$, where the basin is a parabola of revolution, we set $D=1-x^{2}-y^{2}$. Assume that $u_{x}=u_{y}=v_{x}=v_{y}=0$, so that $h_{x x}=h_{y y}=h_{x y}=h_{y x}$ $=0$. Equations (6), (7) and (8) are becomes

$$
\begin{aligned}
& \frac{\partial u}{\partial t}=-g \frac{\partial h}{\partial x}, \quad \frac{\partial v}{\partial t}=-g \frac{\partial h}{\partial y}, \\
& \frac{\partial h}{\partial t}=2(x u+y v)-u \frac{\partial h}{\partial x}-v \frac{\partial h}{\partial y} .
\end{aligned}
$$

\section{B. Numerical Techniques}

A typical finite element with a triangular shape is defined by the local nodes 1, 2, 3 yields the shape of element interpolation function, $\phi_{e}(x, y)$ [7]. Writing a scalar variable as $\phi_{e}=\alpha_{0}+\alpha_{1} x+\alpha_{2} y$. Over any element, $\phi_{1}, \phi_{2}, \phi_{3}$ is a linear function. These are $\phi_{1}=\alpha_{01}+\alpha_{11}+\alpha_{21}, \phi_{2}$ $=\alpha_{02}+\alpha_{12}+\alpha_{22}, \phi_{3}=\alpha_{03}+\alpha_{13}+\alpha_{23}$. Where, $\alpha_{0}, \alpha_{1}$ and $\alpha_{2}$ are constants. In each element, $u$ is written as

$$
\begin{aligned}
& u=u_{1} \phi_{1}+u_{2} \phi_{2}+u_{3} \phi_{3}=u_{1}\left(\alpha_{01}+\alpha_{11}+\alpha_{21}\right) \\
& +u_{2}\left(\alpha_{02}+\alpha_{12}+\alpha_{22}\right)+u_{3}\left(\alpha_{03}+\alpha_{13}+\alpha_{23}\right) \\
& =\sum_{i=1}^{3} u_{i}\left(\alpha_{0 i}+\alpha_{1 i} x+\alpha_{2 i} y\right) .
\end{aligned}
$$

Where, $\phi_{1}, \phi_{2}$ and $\phi_{3}$ are the approximate values for the node numbers 1,2 and 3 . The derivative with respect to $x$ and $y$, are given by

$$
\begin{aligned}
& \frac{\partial u}{\partial x}=u_{1} \frac{\partial \phi_{1}}{\partial x}+u_{2} \frac{\partial \phi_{2}}{\partial x}+u_{3} \frac{\partial \phi_{3}}{\partial x}=\sum_{i=1}^{3} u \frac{y_{j}-y_{k}}{2 \Delta} \\
& \frac{\partial u}{\partial y}=u_{1} \frac{\partial \phi_{1}}{\partial y}+u_{2} \frac{\partial \phi_{2}}{\partial y}+u_{3} \frac{\partial \phi_{3}}{\partial y}=\sum_{i=1}^{3} u \frac{x_{k}-x_{j}}{2 \Delta} .
\end{aligned}
$$

Where, $(i, j, k)=(1,2,3),(2,3,1), \quad(3,1,2) . \Delta$ is the area of the triangle $123, \Delta=\left\{x_{1}\left(y_{2}-y_{3}\right)\right.$ $\left.+x_{j}\left(y_{k}-y_{i}\right)+x_{k}\left(y_{i}-y_{j}\right)\right\} / 2$. In the same way, $v$ and $h$ are written as, $v=v_{1} \phi_{1}+v_{2} \phi_{2}+v_{3} \phi_{3} h=h_{1} \phi_{1}$ $+h_{2} \phi_{2}+h_{3} \phi_{3}$. We have,

$$
\begin{aligned}
& \frac{\partial v}{\partial x}=\sum_{i=1}^{3} v \frac{y_{j}-y_{k}}{2 \Delta}, \frac{\partial v}{\partial y}=\sum_{i=1}^{3} v \frac{x_{k}-x_{j}}{2 \Delta}, \\
& \frac{\partial h}{\partial x}=\sum_{i=1}^{3} h \frac{y_{j}-y_{k}}{2 \Delta}, \frac{\partial h}{\partial y}=\sum_{i=1}^{3} v \frac{x_{k}-x_{j}}{2 \Delta} .
\end{aligned}
$$

At the $i^{\text {th }}$ node, an approximate values of $\partial \mathrm{u} / \partial \mathrm{x}$ and $\partial u / \partial y$ is the average value of the partial derivations over the elements that contain the node, and given by the expressions

$$
\frac{\partial u_{i}}{\partial x}=\frac{1}{m} \sum_{n=1}^{m}\left(\frac{\partial u}{\partial x}\right)_{e_{n}}, \frac{\partial u_{i}}{\partial y}=\frac{1}{m} \sum_{n=1}^{m}\left(\frac{\partial u}{\partial y}\right)_{e_{n}} .
$$


In the same way, the following expressions are obtained for the partial derivatives.

$$
\begin{aligned}
& \frac{\partial v_{i}}{\partial x}=\frac{1}{m} \sum_{n=1}^{m}\left(\frac{\partial v}{\partial y}\right)_{e_{n}}, \frac{\partial v_{i}}{\partial y}=\frac{1}{m} \sum_{n=1}^{m}\left(\frac{\partial v}{\partial y}\right)_{e_{n}}, \\
& \frac{\partial h_{i}}{\partial x}=\frac{1}{m} \sum_{n=1}^{m}\left(\frac{\partial h}{\partial y}\right)_{e_{n}}, \frac{\partial h_{i}}{\partial y}=\frac{1}{m} \sum_{n=1}^{m}\left(\frac{\partial h}{\partial y}\right)_{e_{n}}
\end{aligned}
$$
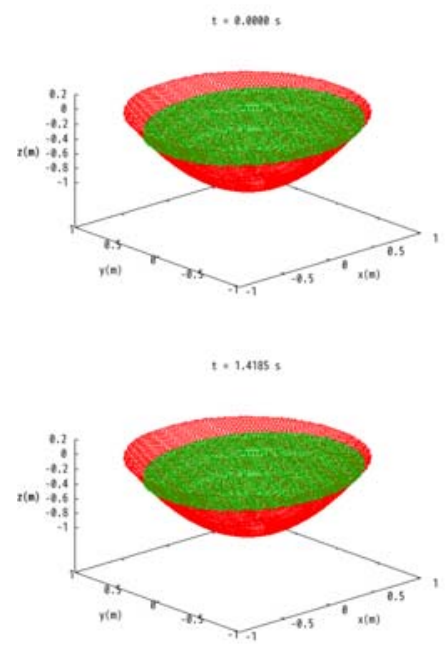

Figure 1. Initial surface of the water and surface of the water at $t=1.4185$ seconds.

The forth-order Adams-Bashforth-Moulton predictor corrector method in PECE mode in conjunction with the Runge-Kutta method was use to solve the following system

$$
\begin{gathered}
\frac{\partial u_{i}}{\partial t}=-g \frac{\partial h_{i}}{\partial x}, \frac{\partial u_{i}}{\partial t}=-g \frac{\partial h_{i}}{\partial y}, \\
\frac{\partial h_{i}}{\partial t}=2\left(x u_{i}+y v_{i}\right)-u_{i} \frac{\partial h_{i}}{\partial x}-v_{i} \frac{\partial h_{i}}{\partial y} .
\end{gathered}
$$

We set $\quad \partial \mathrm{u}_{\mathrm{i}} / \partial \mathrm{x}=\partial \mathrm{u}_{\mathrm{i}} / \partial \mathrm{y}=\partial \mathrm{v}_{\mathrm{i}} / \partial \mathrm{x}=\partial \mathrm{v}_{\mathrm{i}} / \partial \mathrm{y}=0$, $\Delta t=0.0001, g=9.81$ and frequency $\omega=\sqrt{2 g}, \eta=0.05$. Initial conditions are given as $u_{0}=-\eta \omega \sin \left(\omega t_{0}\right), v_{0}$ $=-\eta \omega \cos \left(\omega t_{0}\right), \quad h_{0}=2 \eta \quad\left[\begin{array}{ll}x & \cos \left(\omega t_{0}\right)-y\end{array}\right.$ $\left.\sin \left(\omega t_{0}\right)-0.5 \eta\right]$ and $t_{0}=0.1$ at all nodes.

\section{Numerical Results}

The motion of water was simulated for 1.5 seconds. The water motion resulting from the numerical simulation are shown in the Figure 1. Those are show the initial water surface, and the water surface at $t=1.4185$ seconds. On the other hand, the motion of water surface according to the analytic solution is cyclic with period $2 \pi=\omega t$, since $t=$ $2 \pi / \omega=2 \pi / \sqrt{2 g} \approx 1.4185$ seconds (cf. Figure 2) [6]. The shallow-water wave equations are solved numerically for the water dynamics motion with the moving shoreline.
The comparison between the analytic solution and the numerical solution shows the validity of our numerical techniques.

\section{CALCULATING AREA AND INITIAL CONDITION}

Tsunami wave propagation is simulated over a rectangular region, where the east longitude from $130^{\circ}$ to $140^{\circ}$ and the north latitude from $30^{\circ}$ to $36^{\circ}$. The coordinate system whose origin corresponds to the east longitude $133^{\circ} 30^{\prime}$ and the north latitude $33^{\circ}$ was set and the Gauss-

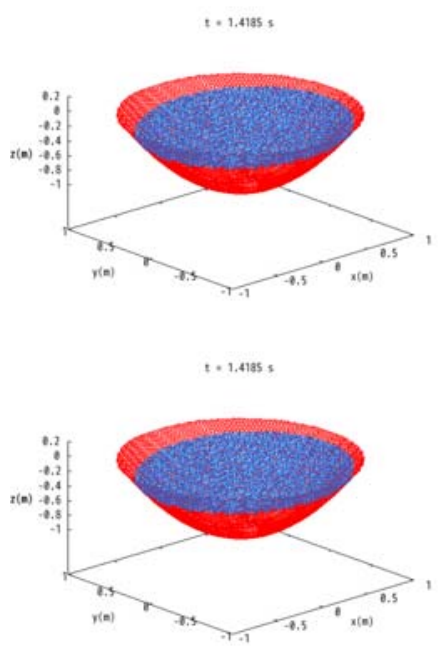

Figure 2. Initial surface of the water and surface of the water at $t=1.4185$ seconds with exact solutions.

Kruger projection was used to convert longitude/latitude to projected coordinate. The interval in the $x$ axis direction was divided into 600 equally spaced intervals. Similarly, the interval in the $y$ axis direction was divided into 360 equally spaced intervals. It consists of 432000 elements and 216961 nodes.

An initial surface displacement based on results by Aida (1972) [8] was set. Initial values of $\zeta_{i}$ were generated, and the initial values of $M_{i}$ and $N_{i}$ were given as $M_{i}=0$, = 0 , when $h_{i}+\varsigma_{i}^{n} \leq 0$, and $\sqrt{M_{i}^{2}+N_{i}^{2}}= \pm \sqrt{g h_{i}}$ when $h_{i}+\varsigma_{i}^{n}>0$.

\section{CONCLUSIONS}

The 1968 Hyuganada tsunami were analyzed using a numerical simulation. The wave height at points near Aburatsu, Hososhima, Saiki and Tosashimizu, respectively. The location of those points are $(\mathrm{N} 1, \mathrm{E} 1)=\left(31.58^{\circ}, 131.42^{\circ}\right)$, $(\mathrm{N} 2, \mathrm{E} 2)=\left(32.43^{\circ}, 131.67 \circ\right),(\mathrm{N} 3, \mathrm{E} 3)=\left(32.9 \circ, 132.2^{\circ}\right)$, $(\mathrm{N} 4, \mathrm{E} 4)=\left(32.68^{\circ}, 133^{\circ}\right)[8]$. Figures $3-7$ show the profiles of wave heights obtained numerically at those four points. Wave records at those points are available for comparison. These results are important and could provide insights for studies in tsunami. 
The tsunami wave propagation is simulated for approximately 3600 seconds from the first main shock of Hyuganada earthquake at 9:42 (JST) on 1 April 1968 with various parameters. (Figures 8-9). Initial surface and the 900 seconds are shown in the Figure 8. Figure 9shows the tsunami wave at the 1800 seconds and 2700 seconds after it was generated. In the future, we will consider the optimization of the element division for simulation.

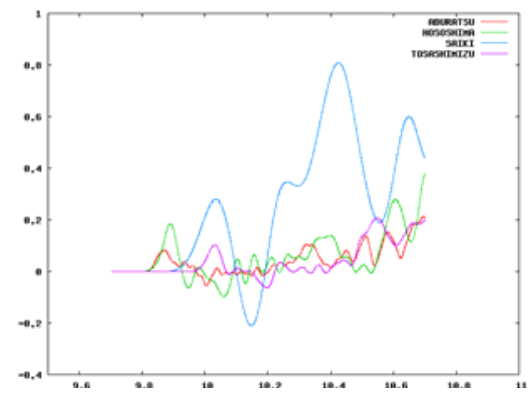

Figure 3. Changes of wave height at the checkpoints.

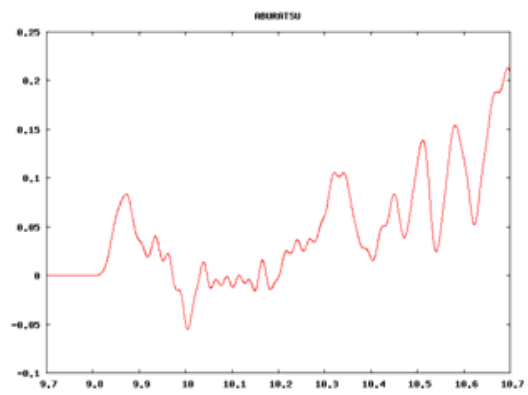

Figure 4. Numerical result for wave height at the point 1 (aburatsu).

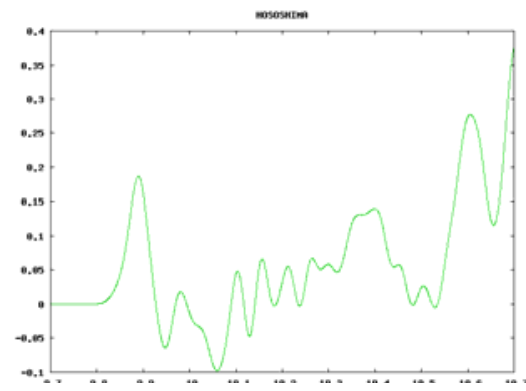

Figure 5. Numerical result for wave height at the point 2 (hososhima).

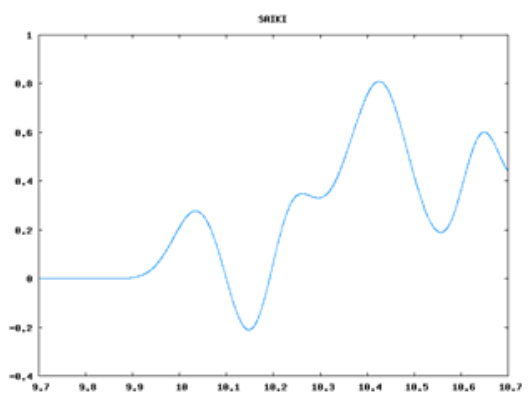

Figure 6. Numerical result for wave height at the point 3 (saiki).

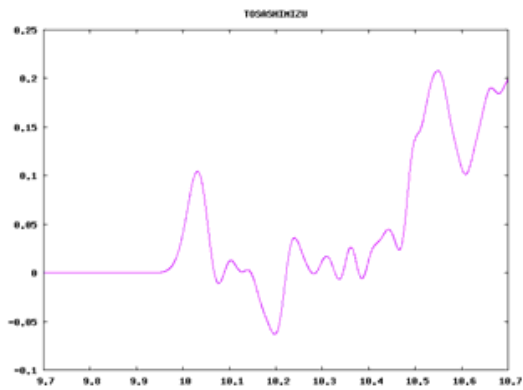

Figure 7. Numerical result for wave height at the point 4 (tosashimizu).
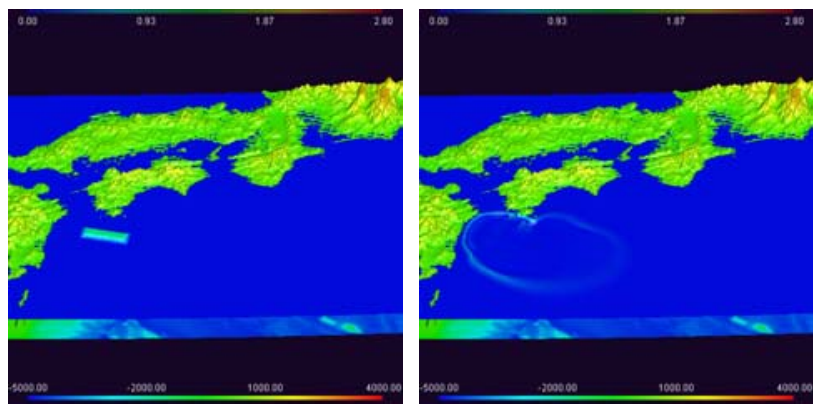

Figure 8. Initial surface of the sea and surface of the sea at 900 seconds.
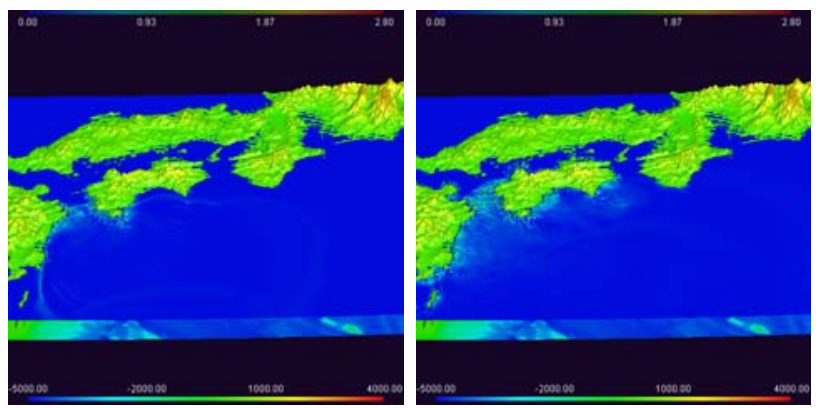

Figure 9. Surface of the sea at 1800 seconds and 2700 seconds. 


\section{REFERENCES}

[1] H. Watanabe, "Japan Tsunami Damage Comprehensive List," 2nd edition, University of Tokyo Press, 1998.

[2] K. H. Lye, "Modeling propagation of 2004 Asian Tsunami: A theoretical Analysis," International Conference of Reservoir Operation and River Management, Guangzhou Three Gorges, China, 17-23 September, 2005.

[3] Kodama, M. Kawahara, "A finite element analysis of shallow water flow with open boundary conditions," Computational Mechanics, Valliappan, Pulmano, Tin-Loi(eds) 1993 Balkema, Rotterdam, ISBN 905410333 7, 11-18, 2003.

[4] M. Kawahara, T. Kodama, and M. Kinoshita, "Finite element method for tsunami wave propagation analysis considering the open boundary conditions," Computer and Mathematics with Applications, 16(1/2), pp. $139-152,1988$.

[5] R. G. Dean and R. A. Dalrymple, "Water Wave Mechanics for Engineers and Scientists," Advanced Series on Ocean Engineering Volume 2, World Scientific, Singapore, 1991.

[6] W. C. Thacker, "Some exact solutions to the nonlinear shallow water wave equations," J. FLUID MECH, Vol. 107, PP. 499-508, 1981.

[7] Society of fluid mechanics numerical, "The flow simulation by finite element method," First Edition, (C) Springer - Verlag., Tokyo, 1998.

[8] I. Aida, "Numerical Estimation of a Tsunami Source, Earthquake," Vol. 25, No. 2, 1972, pp. 343-352. 